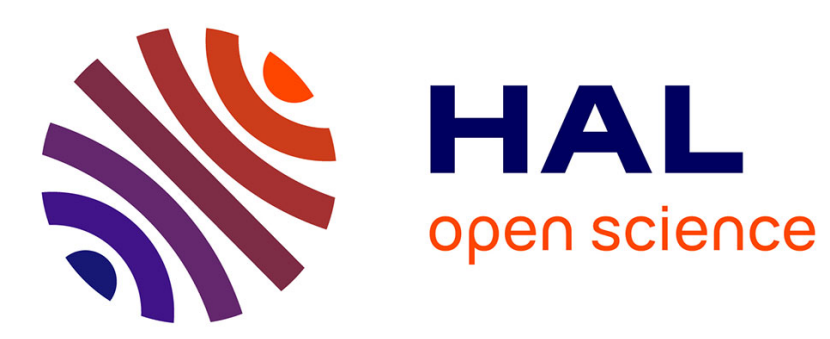

\title{
Étude de réactions (n, n' $\gamma$ ) à l'aide d'un détecteur Ge
} (Li )

\author{
G. Klotz, G. Walter
}

\section{To cite this version:}

G. Klotz, G. Walter. Étude de réactions (n, n' $\gamma$ ) à l'aide d'un détecteur Ge (Li ). Revue de Physique Appliquée, 1969, 4 (2), pp.271-272. 10.1051/rphysap:0196900402027100 . jpa-00243256

\section{HAL Id: jpa-00243256 https://hal.science/jpa-00243256}

Submitted on 1 Jan 1969

HAL is a multi-disciplinary open access archive for the deposit and dissemination of scientific research documents, whether they are published or not. The documents may come from teaching and research institutions in France or abroad, or from public or private research centers.
L'archive ouverte pluridisciplinaire HAL, est destinée au dépôt et à la diffusion de documents scientifiques de niveau recherche, publiés ou non, émanant des établissements d'enseignement et de recherche français ou étrangers, des laboratoires publics ou privés. 


\title{
ÉTUDE DE RÉAGTIONS (n, n' $\gamma$ ) A L'AIDE D’UN DÉTEGTEUR Ge(Li)
}

\author{
G. KLOTZ et G. WALTER, \\ Centre de Recherches Nucléaires, Strasbourg-Cronenbourg.
}

\begin{abstract}
Résumé. - Un spectromètre $\gamma$ de haute résolution utilisant un détecteur $\mathrm{Ge}(\mathrm{Li})$ et destiné à l'étude de la diffusion inélastique de neutrons de $14,5 \mathrm{MeV}$ est décrit. Des résultats préliminaires relatifs à une expérience sur ${ }^{31} \mathrm{P}$ sont présentés.
\end{abstract}

Abstract. - A high resolution $\gamma$-ray spectrometer using a $\mathrm{Ge}(\mathrm{Li})$ detector for $\left(\mathrm{n}, \mathrm{n}^{\prime} \gamma\right)$ measurements at $14.5 \mathrm{MeV}$ bombarding energy, is described. Preliminary results for the ${ }^{31} \mathrm{P}\left(\mathrm{n}, \mathrm{n}^{\prime} \gamma\right)^{31} \mathrm{P}$ reaction are reported.

L'avènement des détecteurs à semiconducteurs $\mathrm{Ge}(\mathrm{Li})$ a provoqué une évolution dans l'étude des réactions $\left(\mathrm{n}, \mathrm{n}^{\prime} \gamma\right)$, à l'aide de la spectrométrie des rayons $\gamma$ émis par les noyaux résiduels. Les techniques antérieures, fondées sur l'utilisation des scintillateurs $\mathrm{NaI}(\mathrm{Tl})$, donnaient une résolution en énergie trop faible pour déterminer la contribution de tous les niveaux en particulier dans le cas de noyaux de nombres de masse $A>20$. Les possibilités d'investigation présentées par la spectrométrie de neutrons par tempsde-vol sont limitées pour les mêmes raisons [1].

Les compteurs $\mathrm{Ge}(\mathrm{Li})$ permettent une mesure plus précise des sections efficaces de production de rayons $\gamma$ et de leur distribution angulaire. Peu de travaux utilisant cette nouvelle technique ont été publiés $[2,3]$. Ils concernent des expériences de diffusion inélastique pour des neutrons d'énergies comprises entre 3 et $8 \mathrm{MeV}$. Le dispositif que nous présentons a été réalisé pour mesurer les sections efficaces de production de rayonnements $\gamma$ émis par des noyaux excités même dans des états rapprochés (écart $\geqslant 20 \mathrm{keV}$ ).

I. Dispositif expérimental ( $f g$. 1). - Le diffuseur (100 à $200 \mathrm{~cm}^{3}$ ) est exposé au bombardement de neutrons issus de la réaction $T(d, n) \alpha$. La détection de la particule associée sert à définir l'instant d'émission et la direction des neutrons susceptibles de diffuser sur les noyaux de l'échantillon. Ce dernier est situé à $1,50 \mathrm{~m}$ de la cible et à une dizaine de centimètres de la diode $\mathrm{Ge}(\mathrm{Li})\left(60 \mathrm{~cm}^{3}\right)$. Cette distance réduite ne permet pas la discrimination $n-\gamma$ par mesure de temps-de-vol entre le diffuseur et le détecteur. L'efficacité de détection de celui-ci et l'épaisseur importante $\mathrm{du}$ blindage nécessaire exigent une telle disposition.

La spectrométrie du rayonnement $\gamma$ se fait à l'aide d'une chaîne d'amplification linéaire à faible bruit associée à un sélecteur d'amplitude (4000 canaux). L'analyse des impulsions est subordonnée à l'enregistrement d'une coïncidence retardée entre la détection

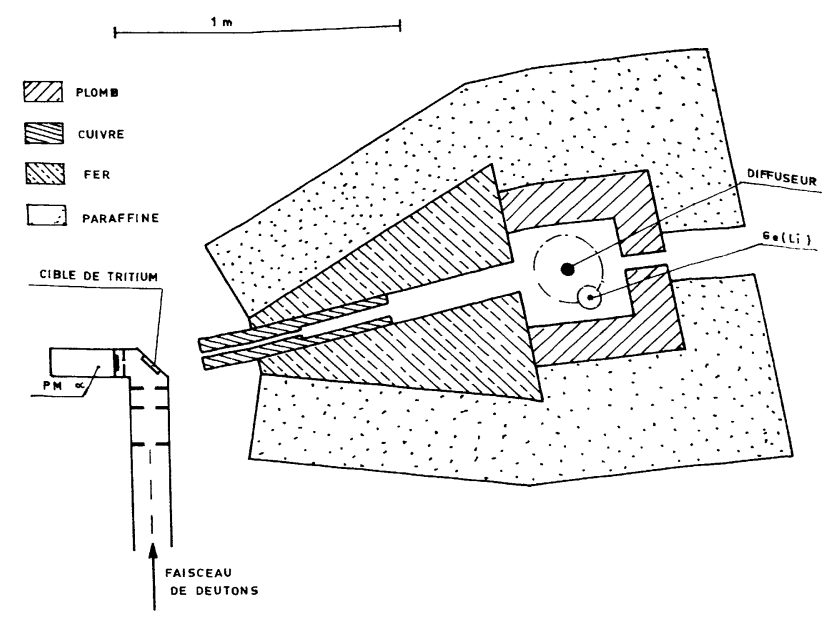

FIG. 1. - Schéma du dispositif expérimental utilisé pour l'étude de réactions $\left(\mathrm{n}, \mathrm{n}^{\prime} \gamma\right)$.

d'une particule $\alpha$ (scintillateur plastique couplé à un tube XP 1021) et celle d'un événement au sein de la diode $\mathrm{Ge}(\mathrm{Li})$. Nous avons atteint une résolution en temps égale à 5 ns pour des énergies de photons comprises entre 0,5 et $10 \mathrm{MeV}$.

Un important blindage de l'ensemble diffuseurdétecteur a permis de réduire le nombre d'impulsions fortuites (environ $20 \%$ des signaux analysés). Le détecteur $\mathrm{Ge}(\mathrm{Li})$, solidaire d'une plaque de fer circulaire mobile sur un chemin de roulement, peut subir un mouvement de rotation autour de l'axe du diffuseur. Ce montage rend possibles des mesures de distributions angulaires entre $20^{\circ}$ et $160^{\circ}$.

La durée d'une expérience, à un angle déterminé, s'étale sur environ 100 heures pendant lesquelles on enregistre la détection de $10^{10}$ particules $\alpha$ pour quelques centaines de milliers d'interactions au sein du diffuseur. 


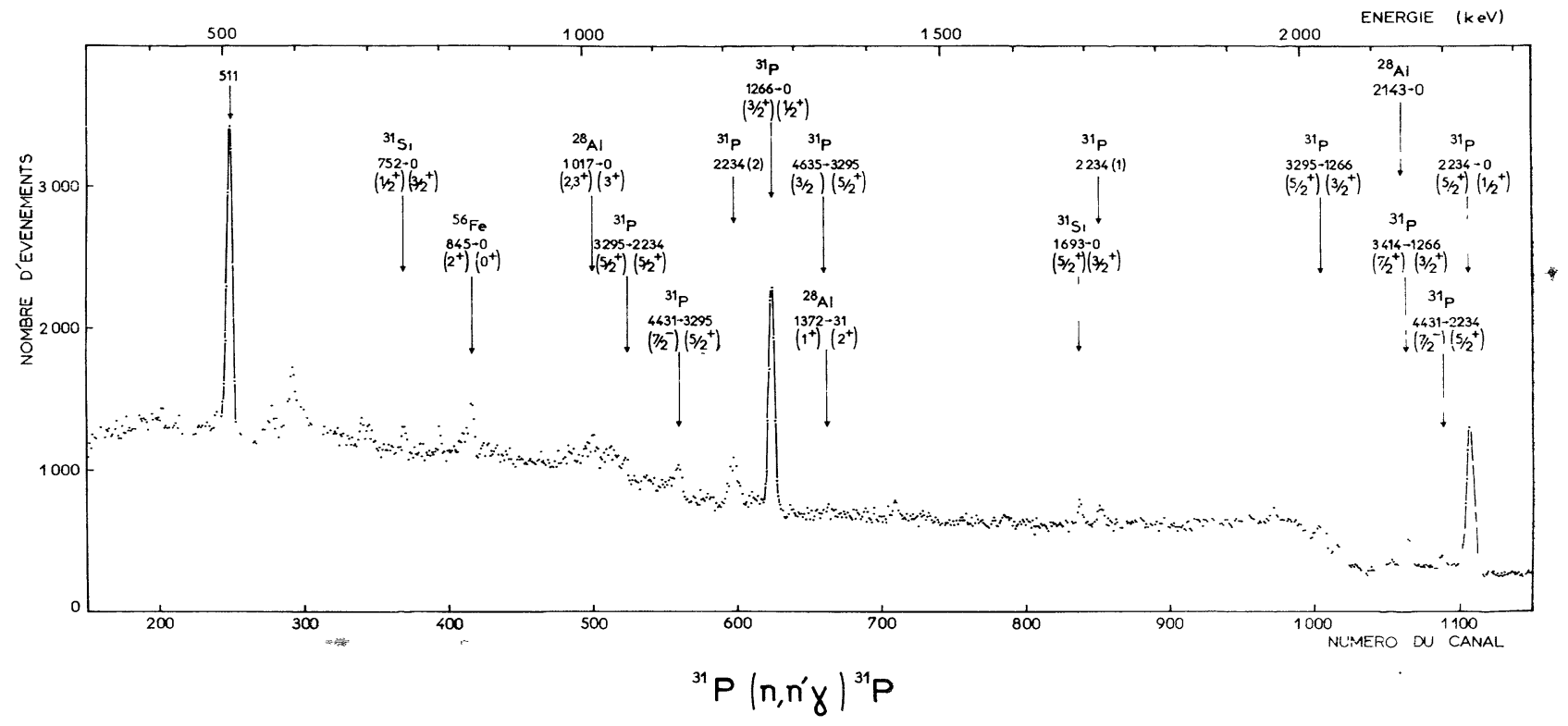

FIG. 2. - Spectre du rayonnement $\gamma$ émis à $90^{\circ}$ par un diffuseur de ${ }^{31} \mathrm{P}$ bombardé par des neutrons de $14,5 \mathrm{MeV}$.

II. Résultats. - Nous avons obtenu une série de spectres concernant la diffusion inélastique de neutrons de 14,5 MeV par les éléments suivants : $\mathrm{Mg}, \mathrm{P}, \mathrm{S}$ et $\mathrm{Cl}$.

Le spectre relatif à ${ }^{31} \mathrm{P}$ est donné à titre d'exemple ( fig. 2). Il rend compte d'un nombre important de transitions faisant intervenir des niveaux jusqu'à $4635 \mathrm{keV}$. Plusieurs raies ont pu être attribuées à la désexcitation des noyaux de recul des réactions $(n, p)$ et $(n, \alpha)$ dans le diffuseur. La différence de section efficace de production observée pour les rayonnements de $1266 \mathrm{keV}$ et de $2234 \mathrm{keV}$ est en accord avec les valeurs des paramètres de déformation nucléaire $\beta_{2}$ déduites des travaux de Levesque [4]. Par suite de la résolution insuffisante des spectromètres de temps-devol de neutrons diffusés, Stelson [1] n'avait pas pu déterminer le coefficient $\beta_{2}$ partiel relatif à l'état $3 / 2^{+}$ (1 $266 \mathrm{keV})$.

Dans tous les spectres, nous observons en outre une distribution continue similaire à celle trouvée par Roturier [5] à l'aide d'un scintillateur $\mathrm{INa}(\mathrm{Tl})$ et attribuée par cet auteur à la désexcitation du noyau composé.

\section{BIBLIOGRAPHIE}

[1] Stelson (P. H.) et al., Nucl. Phys., 1965, 68, 97.

[2] Morgan (I. L.), Texas N.C. Report ORO-2791-19, 1967.

[3] McElisistrem (M. T.) et al., Bull. Am. Phys. Soc., 1967, 12, 1186.

[4] Levengoue (R. J. A.) et al., Phys. Rev., 1963, 132, 1205.

[5] Roturier (J.), Thèse, Bordeaux, 1968. 